\title{
Automatic Subthalamic Nucleus Targeting for Deep Brain Stimulation. A Validation Study
}

\author{
F. Javier Sánchez Castro ${ }^{\mathrm{a}}$, Claudio Pollo ${ }^{\mathrm{a}, \mathrm{b}}$, Jean-Guy Villemure ${ }^{\mathrm{b}}$, \\ Jean-Philippe Thiran ${ }^{\mathrm{a}}$ \\ ${ }^{a}$ École Polytechnique Fédérale de Lausanne (EPFL), Signal Processing Institute, Lausanne, Switzerland \\ ${ }^{\mathrm{b}}$ Department of Neurosurgery, Centre Hospitalier Universitaire Vaudois (CHUV), Lausanne, Switzerland
}

\begin{abstract}
In functional stereotactic neurosurgery, one of the cornerstones upon which the success and the operating time depends is an accurate targeting. The subthalamic nucleus (STN) is the usual target involved when applying Deep Brain Stimulation (DBS) for the Parkinson's disease (PD). Unfortunately, STN is usually not clearly visible in common medical imaging modalities, which justifies the use of atlas-based segmentation techniques to infer the STN location. We propose a scheme that allows both, to perform a comparison between different non-rigid atlas registration algorithms and to evaluate their usability to locate the STN automatically. Using our validationevaluation scheme and accurate registration algorithms we demonstrate that automatic STN localization is possible and accurate.
\end{abstract}

Key words: Non-rigid registration, atlas-based segmentation, deep brain stimulation, surgical planning, pre-operative targeting

\section{Introduction}

A typical DBS procedure starts with the placement of the stereotactic head frame, fixed to the patients skull, that will be used as a coordinate reference. Next an imaging study is taken in order to select the target to stimulate and to plan the trajectories for introducing the electrodes. Usually two kinds of images are taken to be able to visualize different tissues, MR T1-weighted and MR T2-weighted images. The localization of the STN target is performed on coronal T2-weighted images acquired perpendicularly to AC-PC axis and crossing the anterior limit of the Red Nucleus. A study of STN targeting using visible anatomical landmarks can be found in [1]. Then the target coordinates are reported to the T1-weighted image where trajectories are planned. Once in the operating room the head frame is fixed to the operating

Email address: francisco.sanchezcastro@epfl.ch (F. Javier Sánchez Castro). 
table, a small hole is drilled into the patient's skull according to pre-operative trajectories. Because of the difficulty to see the STN directly from medical images, the pre-operative target is only an estimation of the real location. The electrode's location has to be adjusted intra-operatively using electrophysiological recordings and from stimulation tests.

The goal of the study reported in this paper is to sound the possibility of automatically locate the STN using existing registration techniques and to compare their performance and usability. Knowing the location of the STN for all our patient's database, a patient is chosen as atlas and each individual patient mapped back to the atlas using the registration methods considered in this study to obtain an estimation of the STN which is compared to the real location given by the expert.

\section{Material and Methods}

\subsection{Data}

For each patient of our bilaterally implanted parkinsonian patient's database two kinds of images were taken pre-operatively: a 3D T1-weighted magnetization-prepared rapid acquisition gradient echo (MPRAGE) MRI sequence (Siemens Vision ${ }^{\circledR}, 1.5$ T, Erlangen, Germany) TR 9.7 ms, TE 4 ms, number of slices/slice thickness: $164 / 1.40 \mathrm{~mm}$, FOV 280x280, matrix 256x256, pixel size 1.09x1.09 mm, and few coronal slices (due to the time taken for this kind of imaging sequence) of an inversion recovery (IR) T2weighted, TR 2,560 ms, TE 4 ms,number of slices/slice thickness: 7/3 mm, FOV 300x300, matrix 512x512, pixel size $0.59 \times 0.59 \mathrm{~mm}$. Taking profit from the fact that in some rare cases the STN is visible in MR T2-weighted images, a reference can be constructed and used as a ground truth. To do this neurosurgeon experts have been asked to select patients with clearly visible STN in MR T2-weighted images amongst our patient's database. After exhaustive inspection 8 patients were selected (16 STNs).

\subsection{Validation Scheme}

An experimented neurosurgeon with wide experience in PD surgery and targeting has been asked to click the target point (2 STN) for each selected T2 series. This process has been repeated 5 times for each patient at different days to avoid that the expert be influenced by previous targeting choices. With this data we were able to compute a statistical mean target point coordinates which we call the real targets. Amongst the 8 subjects selected, the one with the most clearly visible STN has been selected as a reference subject, both for the right and left sides. Intra-expert variability statistics can also be extracted. Using these data we can consider our reference subjects as an atlas. Then, by non-rigidly registering the atlas with the patient image, we obtain an estimation of the STN position in the patient. The procedure is as follows. The first step is, for each patient, to report its MR T2 coordinates to MR T1 coordinates to obtain the location of the real targets in the $\mathrm{T} 1$ domain. To do this a rigid registration between the two modalities (6 degrees of freedom: translation and rotation) has been performed [2]. The next step consists in applying each of the registration algorithms under study to get an estimation of the position of the STN. This estimation is obtained by registering the MR T1-weighted image of the atlas with the MR T1-weighted image of the patient under study. In this work four methods were considered (see section 2.3). Finally, repeating this for the 7 datasets (14 STNs) involved in this study, Euclidean distances from estimated to real targets (seen as the mean target point given by the expert) for each STN are calculated and statistics are extracted to evaluate the performance of the different methods. 


\subsection{AC-PC Targeting and Registration Algorithms}

To face up to image registration the following methods have been tested:

- Atlas-based (AC-PC) targeting. AC-PC referential together with brain atlases is one of the methods used to target the STN in medical environment, mainly when STN is not clearly visible in MR T2-weighted images which is the usual case. Neurosurgeons have to locate the anterior and posterior commissures (AC-PC points). Then, using a stereotaxic atlas and taking as the origin the midcommissural point (MCP), we can estimate that the STNs are located at coordinates (following Schaltenbrand-Wahren [3]): anteroposterior (AP) $-3 m m$, lateral (LAT) $\pm 12 m m$ (left and right side) and vertical (VERT) $-4 m m$.

- Affine registration. It is an independent implementation based on the work of Maes et al. [2]. The 12 parameters are optimized in order to maximize the mutual information between the images to be registered [4]. Affine registration is also used as a pre-alignment step for non-rigid transformations described below.

- Demons algorithm. It is an independent implementation of the intensity-based algorithm developed by Thirion which uses the Maxwell's demons concept [5].

- B-splines algorithm. It is a mutual-information-based free-form deformation algorithm which displacement field is modelled as a linear combination of B-splines lying in a regular grid (uniformly spaced control points). See [6] as a reference of registration using splines.

\section{Results}

\subsection{Target Selection and Surgeon Variability}

Two main statistics can be extracted from the data given by the expert: intra-surgeon variability and the mean target point coordinates for each STN which will be used as a real target coordinates.

In order to evaluate the repeatability of the neurosurgeon targeting we have proceeded in different manners. First we have computed, for each STN, the Euclidean distance between each couple of neurosurgeon's clicks (5 clicks per STN, 10 distances per STN, 160 distances for the eight selected patients from the database) and we obtain a mean and a standard deviation (using the unbiased estimator) of $1.67 \pm 0.98 \mathrm{~mm}$, which we call paired variability. The same information can be extracted computing the centroid for each STN and calculating the Euclidean distances to each click. This gives a mean and unbiased standard deviation of $1.06 \pm 0.61 \mathrm{~mm}$, which we call centroid variability. These quantities allow us to get an idea of the surgeon variability and its accuracy when clicking over the pre-operative target. If we only consider to the STNs used as a reference we obtain a paired variability of $1.64 \pm 0.88 \mathrm{~mm}$ and $1.18 \pm 0.52 \mathrm{~mm}$, a centroid variability of $1.10 \pm 0.32 \mathrm{~mm}$ and $0.75 \pm 0.38 \mathrm{~mm}$ and a mean distance to the MCP of $12.38 \pm 0.61 \mathrm{~mm}$ and $13.63 \pm 0.54 \mathrm{~mm}$ for the left and right sides respectively. In figure 1 the points targeted by the expert for the left (figures 1(a), 1(b), 1(c)) and right (figures 1(d), 1(e), 1(f)) STNs chosen as a reference can be seen. For visualization purposes, each point is projected onto the three orthogonal planes passing through the centroid and showed using a circle (radius of $1 \mathrm{~mm}$ ).

We can also decompose these distances in anteroposterior (AP), lateral (LAT) and vertical (VERT) coordinates which allows comparing directly the mean STN location for our database with usual STN coordinates from the atlases (e.g. [3]). In table 1 we show the mean and standard deviation of the coordinates referred to the MCP for the 16 STNs used in this study as well as their mean distance to the $\mathrm{MCP}$. 


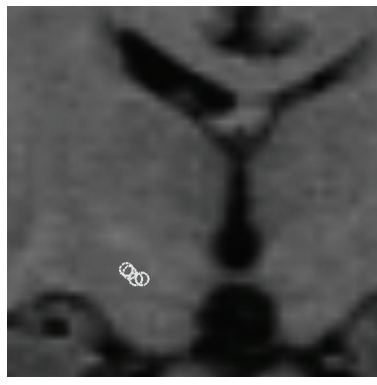

(a) L-STN Coronal.

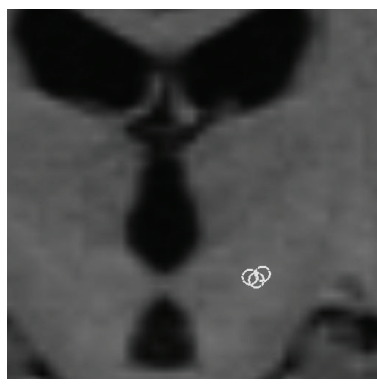

(d) R-STN Coronal.

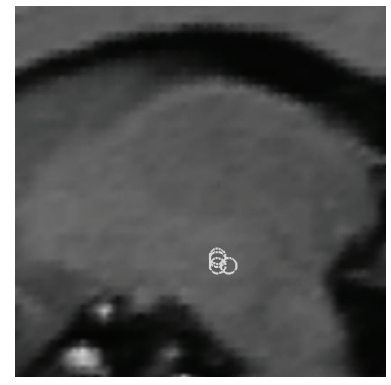

(b) L-STN Sagital.

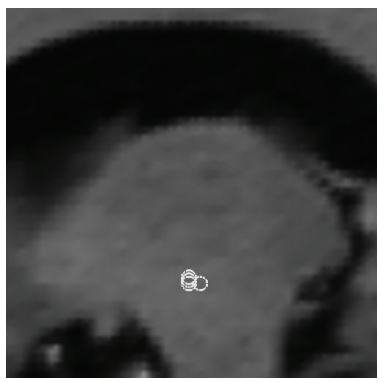

(e) R-STN Sagital.

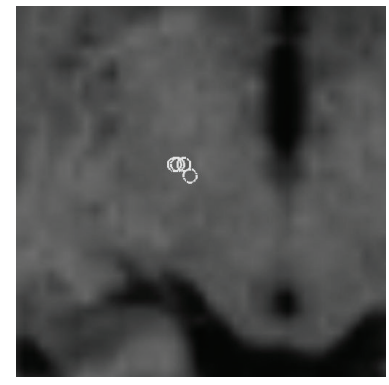

(c) L-STN Axial.

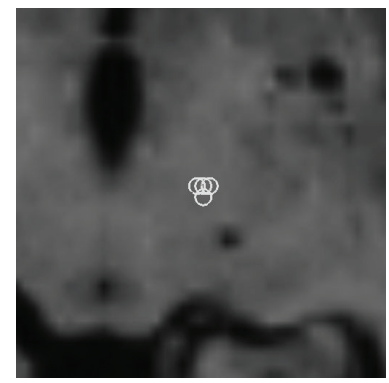

(f) R-STN Axial.

Fig. 1. Reference STN expert targeting.

Table 1

\begin{tabular}{cc}
\hline Coordinates & mean \pm std \\
\hline AP & $-3.29 \pm 1.36 \mathrm{~mm}$ \\
\hline LAT & $\pm 12.57 \pm 1.39 \mathrm{~mm}$ \\
\hline VERT & $-3.16 \pm 0.89 \mathrm{~mm}$ \\
\hline MCP-distance & $13.47 \pm 1.37 \mathrm{~mm}$ \\
\hline
\end{tabular}

Mean STN coordinates referred to the MCP.

\subsection{Target Estimation and Assessment of the Methods}

The statistics (mean and unbiased standard deviation) of the errors committed when applying these algorithms and methods to locate the STN as described in 2.3 are shown in table 2. In order to compare the results statistical tests have been done. In figure 2 a statistical box plot for the different methods tested is showed. A paired T-test of the hypothesis that the errors come from distributions with equal means has been performed over each pair of methods at a $5 \%$ significance level. We obtain that the errors committed with B-splines, demons and AC-PC based methods are not significantly different. However the results show that affine registration is significantly different from the other methods ( $p$ - values of $0.0469,0.0270$, and 0.0329 for the B-splines, demons and AC-PC methods respectively).

In figure 3 we show the projection of each STN estimation (in white) onto the reference subject (in red) using the B-splines registration algorithm. Each point is represented by a circle of $1 \mathrm{~mm}$ of radius whose coordinates are projected onto the reference subject point coordinates (in red) in order to visualize the points in each view and referred to the reference subject point. The estimated targets are located very close to the real target and forming tight clouds of points showing that this kind of automatic estimation 
Table 2

\begin{tabular}{cc}
\hline Methods & mean \pm std \\
\hline Affine & $2.65 \pm 1.25 \mathrm{~mm}$ \\
\hline Demons & $1.97 \pm 0.85 \mathrm{~mm}$ \\
\hline B-Splines & $1.80 \pm 0.62 \mathrm{~mm}$ \\
\hline AC-PC & $1.98 \pm 0.81 \mathrm{~mm}$ \\
\hline
\end{tabular}

Estimation Errors

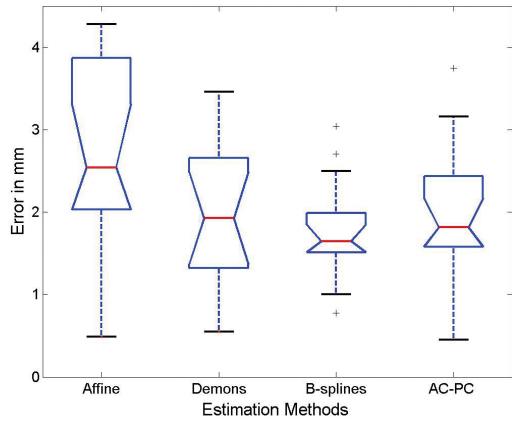

Fig. 2. Statistical box plot of the errors committed using different methods.

is reliable and well suited for this application.

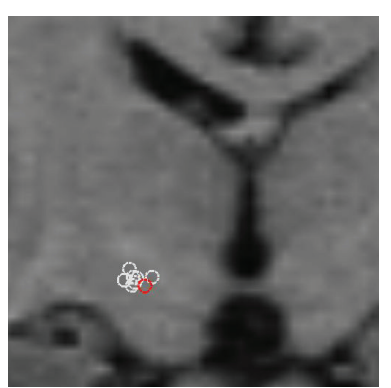

(a) L-STN Coronal.

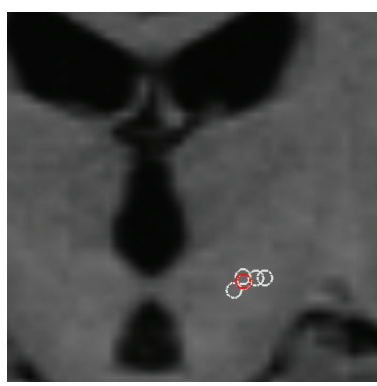

(d) R-STN Coronal.

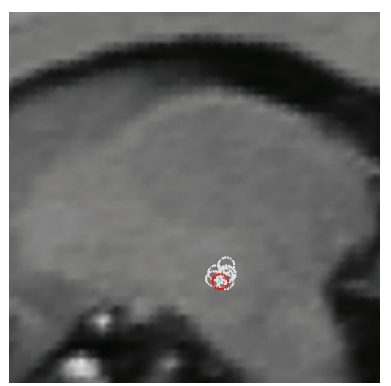

(b) L-STN Sagital.

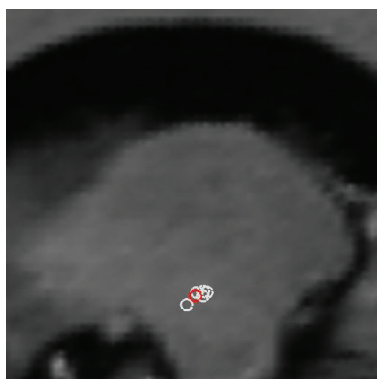

(e) R-STN Sagital.

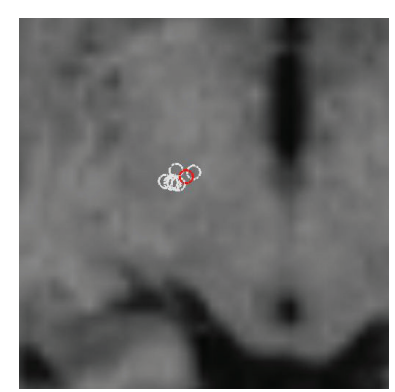

(c) L-STN Axial.

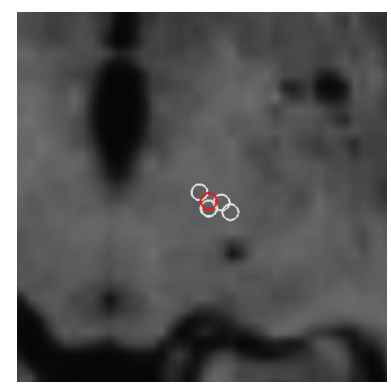

(f) R-STN Axial.

Fig. 3. STN estimation using B-splines. 


\section{Discussion and Conclusions}

The main conclusion one can extract from the results we have obtained from this evaluation-validation work is that automatic STN location is possible and accurate. As we can see, by simple inspection of the numerical results, the B-splines method shows the best performance with the smallest mean error and adjusted (unbiased) standard deviation but closely followed by demons and AC-PC methods. The points project on tight clusters showing the robustness of this kind of estimation method. The statistical tests have shown that global affine registration is not enough for our application and that there are not significant differences between the errors committed using the B-splines, Demons or AC-PC referential-based techniques. Although AC-PC referential-based method shows an acceptable performance its estimation needs AC and PC point's identification by an expert and does not take into account inter-patient variability, which is very important at a single patient level. The fact that AC-PC shows such a good behavior can be explained because the selected patients follow well the average brain defined by the atlas. But what about patients with abnormal anatomy or damaged brains? Although the choice of the STN of reference can influence the results, the methods have been tested using 6 different combinations of STN-pairs (left and right references) and the results were similar. The automatic estimation of the STN can be also used as a first and fast pre-operative target estimation that can be refined by the neurosurgeon criterion. As our patient's database increases and more experts included in the study, we will be able to establish stronger assessments and conclusions.

\section{Acknowledgements}

This work is supported by the Swiss National Science Foundation under grant number 205320-101621.

\section{References}

[1] C. Pollo, R. Meuli, P. Maeder, F. Vingerhoets, J. Ghika, J-G. Villemure, "Subthalamic Nucleus Deep Brain Stimulation for Parkinson's Disease: Magnetic Resonance Imaging Targeting Using Visible Anatomical Landmarks," Proceedings of the Meeting of the American Society for Stereotactic and Functional Surgery, New York, vol. 80, no. 1-4, 2003.

[2] F. Maes, A. Collignon, D. Vandermeulen, G. Marchal, P. Suetens, "Multimodality image registration by maximization of mutual information," IEEE transactions on Medical Imaging, vol. 16, no. 2, pp. 187-198, April 1997.

[3] G. Schaltenbrand, W. Wahren, "Atlas for Stereotaxy of the Human Brain," ed. 2, Stuttgart, Thieme, 1977.

[4] P. Viola, "Alignment by maximization of Mutual Information," M.I.T. Artificial Intelligence Technical Report, no. 1548, June 1995.

[5] J.-P. Thirion, "Image matching as a diffusion process: an analogy with Maxwells demons," Medical Image Analysis, vol. 2, no. 3, pp. 243-260, 1998.

[6] D. Rueckert, L. I. Sonoda, C. Hayes, D. L. G. Hill, M. O. Leach, D. J. Hawkes, "Nonrigid registration using Free-Form Deformations: Application to breast MR images," IEEE Transactions on Medical Imaging, vol. 18, no. 8, pp. 712-721, 1999. 